\title{
Editorial preface for special issue of FAIM 2015 published in IJAMT
}

\author{
Chike F. Oduoza ${ }^{1}$
}

Published online: 23 June 2017

(C) Springer-Verlag London Ltd. 2017

The International Conference on Flexible Automation and Intelligent Manufacturing (FAIM) is the leading international forum for dissemination of current research on all aspects of automation and manufacturing, and highlights how theoretical models and principles underpin practice in a production environment. The conference will link researchers and practitioners both from industry and academia specializing in automation, manufacturing and other related engineering disciplines from around the world.

The 25th FAIM conference (silver anniversary of FAIM) was aimed to build on the success of earlier conferences held annually at various locations around the world. The list of previous FAIM conferences by year and location are as follows:

1. 1991 University of Limerick, Limerick, Ireland

2. 1992 Virginia Tech (in Washington DC), USA

3. 1993 University of Limerick, Limerick, Ireland

4. 1994 Virginia Tech, Blacksburg, Virginia, USA

5. 1995 University of Stuttgart, Germany

6. 1996 Georgia Institute of Technology, Atlanta, USA

7. 1997 University of Teesside, Middlesbrough, UK

8. 1998 Portland State University, Portland, Oregon, USA

9. 1999 Tilburg University, Tilburg, Netherlands

10. 2000 University of Maryland, Maryland, USA

11. 2001 Dublin City University, Dublin, Ireland

Chike F. Oduoza

C.F.Oduoza@wlv.ac.uk

1 Process and Manufacturing Engineering, University of Wolverhampton, Wolverhampton WV1 1LY, UK
12. 2002 Dresden University of Technology, Dresden, Germany

13. 2003 University of South Florida, Tampa, USA

14. 2004 Ryerson University, Toronto, Canada

15. 2005 University of Duesto, Bilbao, Spain

16. 2006 University of Limerick, Limerick, Ireland

17. 2007 Penn State Great Valley, Philadelphia, USA

18. 2008 University of Skövde, Sweden

19. 2009 University of Teesside, UK

20. 2010 University of California, San Francisco, USA

21. 2011 Fen Cheng University Taiwan

22. 2012 Tampere University of Technology

23. 2013 University of Porto, Portugal

24. 2014 University of Texas, San Antonio, USA

The focus of the conference hosted by the Faculty of Science and Engineering, University of Wolverhampton, West Midlands, UK, was to address the theme:

Designing for Advanced, High Value Manufacturing and Intelligent Systems for the 21st Century. FAIM 2015 conference covered a wide range of topics including, but not limited to, the following:

- Additive layer manufacturing

- Advanced automotive and aerospace manufacturing

- Computer-aided design/computer-aided manufacture/ flexible manufacturing

- Engineering for sustainability, education and training

- Enterprise knowledge management

- Industrial engineering and operations management

- Lean and agile manufacturing

- Manufacturing ergonomics and human factors

- Manufacturing operations management and optimisation 
- Manufacturing processes and technology

- Manufacturing technology and intelligent systems

- Modelling and simulation

- Product design and manufacture

- Production planning and scheduling

- Product, process and factory integrated design

- Engineering and risk management systems in production and construction environment

- Robotics and Computer-Integrated Manufacturing

- SMEs competitiveness and smart factory

- Supply chain and logistics

- Technologically complex and innovative products and advanced materials

- Technology management and innovation

FAIM is a reputable international meeting held annually at selected Universities around the world; last year, it was held at Chonbuk National University, South Korea.

During FAIM 2015, over 200 abstracts were received from many countries; (USA, UK, Germany, France, China, South Korea, Israel, Ireland, Czech Republic, Iran, Mongolia, Egypt, Turkey, South Africa, Nigeria, Brazil, Spain, India, Croatia, Sweden, Finland, Italy, Pakistan, Portugal, Taiwan, Canada and India) which demonstrate the reputation of this international meeting. Two volumes of the conference proceedings with a total of 1204 pages were produced and published by The Choir Press (ISBN978-1-910864-00-5 for volume 1 and ISBN978-1-910864-01-2 for volume 2).

One hundred fifty delegates from 32 countries presented papers over the 3 days of the conference and out of the collection; forty papers from across the conference themes were carefully selected and subjected to rigorous peer review process. Overall, fifteen papers were recommended for publication in Robotics and Computer-Integrated Manufacturing (RCIM) while seven papers were accepted for publication in International Journal for Advanced Manufacturing Technology (IJAMT). We thank all the authors for their patience during the lengthy and rigorous editorial process.

The support received from the International Scientific Committee in reviewing manuscripts are gratefully acknowledged. We also wish to thank the key note and plenary speakers, panellists and all authors for their valuable contribution to the success of this event. Altogether, they have highlighted the state of the art on designing for advanced high value manufacturing and also created an awareness of the future direction of manufacturing technology and intelligent systems. We also wish to recognize the founders of FAIM conference which commenced over 25 years ago, Professor W.G Sullivan and Professor M.M Ahmad for their guidance of this reputable, annual, international academic research meeting.

I hope you enjoy this collection of high-value research papers.
Professor Chike F Oduoza, CEng, FIChemE, Guest Editor, Special Issue of FAIM 2015 in RCIM

Professor in Process and Manufacturing Engineering, University of Wolverhampton, WV11LY, UK.

Email address: C.F.Oduoza@wlv.ac.uk

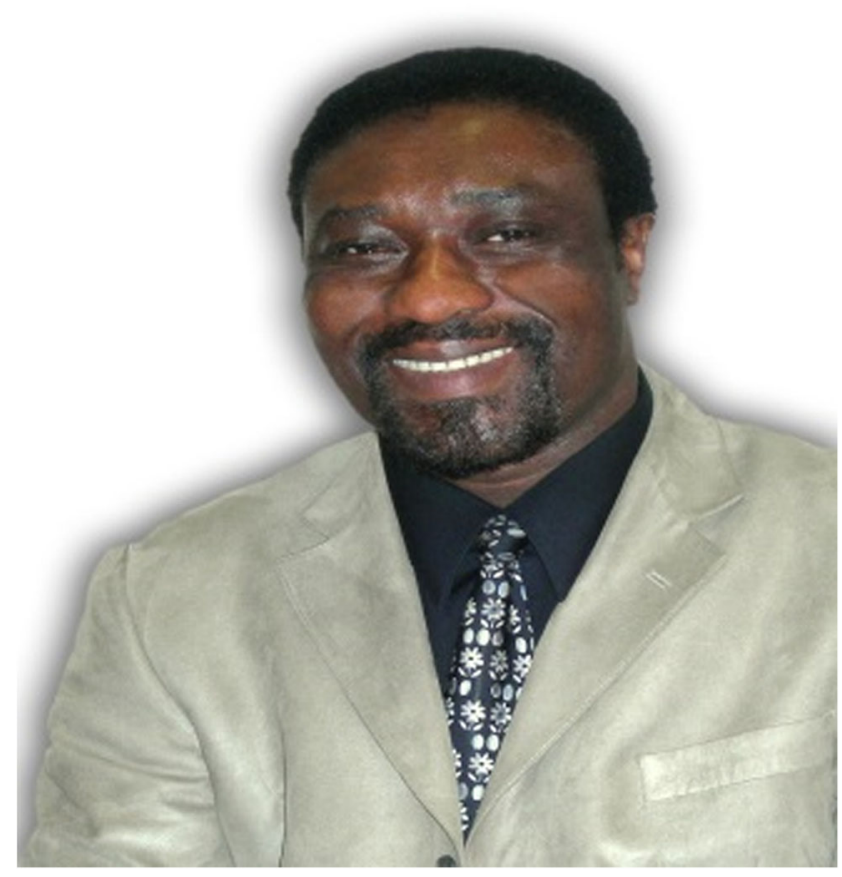

Highlights of the papers included in this special issue of IJAMT This special issue of IJAMT captures outstanding papers presented at Flexible Automation and Intelligent Manufacturing Conference (FAIM) 2015 as follows.

Schmidt and Wang studied cloud-enhanced predictive maintenance and proposed a framework and methodology to handle and process maintenance, production and factoryrelated data from the first lifecycle phase to the operation and maintenance phase with a view to validate the work in the context of real industrial applications.

Li-Chih Wang, Allen Wang, Chun-Ya Chueh developed capacity analysis and planning simulation model for semiconductor fabrication. The outcome was the development of an object-oriented capacity analysis and planning simulation (CAPS) model which takes into account the production constraints, the operation characteristics of machine tools and the dispatching rules applied in a full scale water fab.

Vosniakos and Konstantinou researched on the rough-cut fast numerical investigation of temperature fields in selective laser sintering/melting and investigated selective laser sintering/melting (SLS/SLM) processes by a thermal model solved with a finite difference scheme. They developed a model that can be used to obtain a preliminary picture of the response of any material to SLS/SLM processing provided that the appropriate input data is available. 
Li, Dauod, Sang Won Yoon and Srihari studied multiobjective optimization of the order scheduling problem in mail-order pharmacy. The outcome was a fully dedicated machine environment with $80 \%$ more total collation delays as compared to a fully flexible machine environment and 25\% more total collation delays as compared to a multi-purpose machine environment. They also confirmed that genetic algorithm can achieve the optimal make span in most cases, while minimizing the total collation delays by $96 \%$ when compared to least processing time and least total workload.

Flynn, Shokrani, Vichare, Dhokia and Newman developed a methodology for identifying location errors in 5-axis machine tools using a single ballbar set-up. They developed a single set-up testing method to identify all rotary axis locations errors, whilst remaining robust in the presence of set-up error and linear axis squareness error. Using the VMT, error identification residuals were found to be $2.7 \%$, or less and all errors can be measured using a single set-up, and values are sufficiently close to the values measured using conventional multi-set-up procedures for compensation. This approach will significantly reduce set-up durations and remove the need for any modified testing hardware.

Sormaz and Patel described the development and evaluation of feature-focused dynamic routing policy. The dynamic policy is based on integrative methodology in which process planning system IM planner is integrated with FMS simulation module. The proposed framework was evaluated in an experimental FMS simulation module, implemented in arena, in which two performance criteria, machine utilization and WIP were used to make routing decisions.

Nemer and Konukseven studied off-line nominal path generation of 6-DoF robotic manipulator for edge finishing and inspection processes. The focus of the study was to generate an efficient, collision-free, nominal tool path needed for edge finishing and inspection by utilizing a 6-DoF robotic arm. The overall nominal tool path involves analysing the geometry of the workpiece, determining and designing efficient collisionfree tool path and finally generating the tool path data for the robot and then verifying it. This package significantly simplifies the complexity of planning an efficient path for performing edge finishing and inspection. 